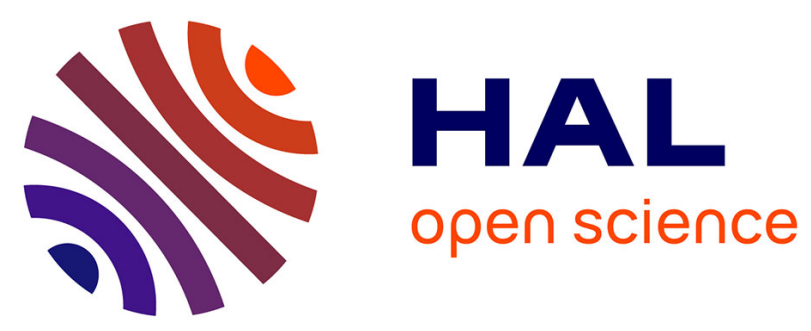

\title{
Comportement alimentaire des séniors: impact des différents facteurs de santé orale sur les dimensions de l'acte alimentaire
}

Mathilde Vandenberghe-Descamps, Hélène Labouré, Chantal Septier, Aurélie

Prot, Carole Tournier, Evelyne Vigneau, Gilles Feron, Claire Sulmont-Rossé

\section{To cite this version:}

Mathilde Vandenberghe-Descamps, Hélène Labouré, Chantal Septier, Aurélie Prot, Carole Tournier, et al.. Comportement alimentaire des séniors: impact des différents facteurs de santé orale sur les dimensions de l'acte alimentaire. JFN 2017, Journées Francophones de Nutrition, Dec 2017, Nantes, France. 1 p., 2017. hal-02790573

\section{HAL Id: hal-02790573 \\ https://hal.inrae.fr/hal-02790573}

Submitted on 5 Jun 2020

HAL is a multi-disciplinary open access archive for the deposit and dissemination of scientific research documents, whether they are published or not. The documents may come from teaching and research institutions in France or abroad, or from public or private research centers.
L'archive ouverte pluridisciplinaire HAL, est destinée au dépôt et à la diffusion de documents scientifiques de niveau recherche, publiés ou non, émanant des établissements d'enseignement et de recherche français ou étrangers, des laboratoires publics ou privés. 


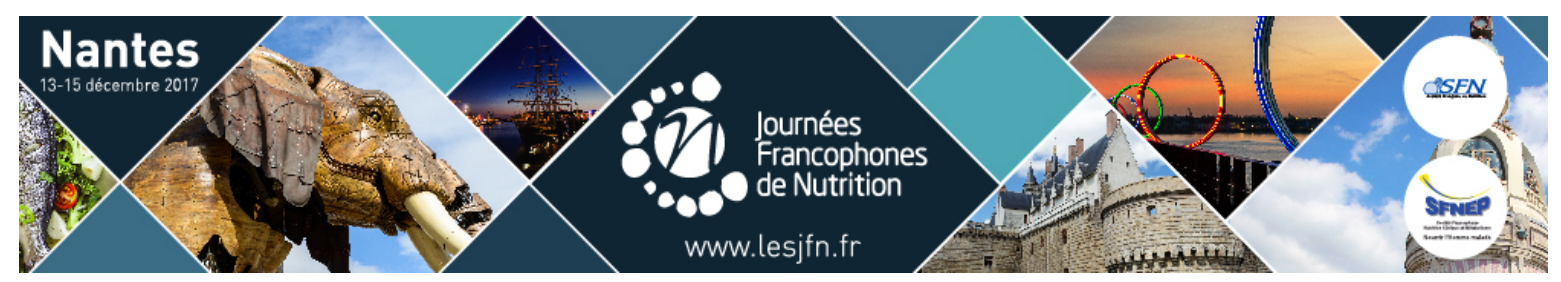

Comportement alimentaire - Fonction cérébrale

JFN2017/1370

\section{Comportement alimentaire des séniors : impact des différents facteurs de santé orale sur les dimensions de l'acte alimentaire}

Mathilde Vandenberghe-Descamps ${ }^{*}$, Hélène Labouré ${ }^{1}$, Chantal Septier ${ }^{1}$, Aurélie Prot $^{1}$, Carole Tournier ${ }^{1}$, Evelyne Vigneau ${ }^{2}$, Gilles Feron ${ }^{1}$, Claire Sulmont-Rossé ${ }^{1}$

${ }^{1}$ Centre des Sciences du Goût et de l'Alimentation, AgroSup Dijon, CNRS, INRA, Univ. Bourgogne Franche-Comté, Dijon, ${ }^{2}$ StatSC, Oniris, INRA, Nantes, France

\section{Discipline: Expérimental/mécanismes cellulaires et moléculaires}

Présentation préférée: Communication orale

Introduction et but de l'étude: Chez l'homme, la mise en bouche d'un aliment est le début du processus de dégradation et de digestion. Avec l'âge, la santé orale évolue. Un aliment difficile à mâcher, à humidifier ou avaler rend l'acte alimentaire difficile. L'objectif de cette étude est de déterminer le rôle des différents facteurs de santé orale (état dentaire, flux salivaire, force musculaire) sur les dimensions physiologiques (faculté à former un bol alimentaire, libération et perception de la flaveur) et/ou sur la prise alimentaire et la corpulence des séniors.

Matériel et méthodes: 61 séniors ( $>65$ ans) vivant à domicile et ne présentant pas de pathologie chronique ont été recrutés (âge $=$ $72 \pm 5 ; 31 \mathrm{~F} ; 30 \mathrm{H})$ et caractérisés sur la base des dimensions suivantes :

Santé orale via un examen clinique (flux salivaire au repos et stimulé, viscosité salivaire, nombre de dents, nombre d'unités fonctionnelles, force musculaire de la langue)

- $\quad$ Auto-évaluation de la santé orale (GOHAI, xérostomie)

- $\quad$ Perceptions sensorielles (détection des saveurs et des odeurs)

- $\quad$ Mécanismes en bouche (faculté à former un bol de carotte, libération d'arômes)

- $\quad$ Variables nutritionnelles (enquête alimentaire de 5 jours)

- $\quad$ Corpulence (impédance, IMC)

Les analyses statistiques ont été faites par Partial Least Square-Path Modeling. Le protocole a été approuvé par le CPP Est-1 N IRB 2016-A00916-45.

Résultats et Analyse statistique : Les résultats montrent que les personnes qui ont un flux salivaire élevé libèrent davantage d'arômes en bouche $(r=0.27 ; p<0.05)$ et consomment plus de calories et de protéines que les autres $(r=0.31 ; p<0.05)$. Les personnes ayant une bonne santé dentaire s'estiment en meilleure santé orale et ont une meilleure faculté que les autres à former un bol de carotte déglutissable $(r=0.51 ; p<0.001$ et $r=0.56 ; p<0.01$ respectivement). En revanche, l'état dentaire n'influence pas les prises énergétique et protéique. Enfin, les personnes ayant une faible force musculaire de la langue ont une consommation énergétique et protéique plus élevée que les autres $(\mathrm{r}=-0.18 ; \mathrm{p}<0.05)$. De plus, une meilleure perception des composés de la flaveur diminue la consommation d'énergie et de protéines $(\mathrm{r}=-0.40 ; \mathrm{p}<0.01)$. Finalement, plus les prises calorique et protéique sont élevées, plus la corpulence des personnes âgées est élevée $(\mathrm{r}=0.35 ; \mathrm{p}<0.01)$.

Conclusion: Cette étude montre que les facteurs de santé orale (dentition, salivation, force musculaire) jouent des rôles différents dans les processus de mastication et de prise alimentaire chez les personnes âgées. Ces résultats permettront de développer une offre alimentaire adaptée aux différents comportements alimentaires induits par les troubles oraux survenant avec l'âge.

Remerciements: Projet financé par l'ANR (AlimaSSenS ANR14CE20000301)

Conflits d'intérêts: Aucun conflit à déclarer 